\title{
Multi-language Online Word Processor for Learners and the Visually Impaired
}

\author{
Shiblee Imtiaz Hasan \\ Department of Computer Science and Engineering, BRAC University, \\ 66, Mohakhali C/A, Dhaka 1212, Bangladesh \\ shiblee@ieee.org
}

\begin{abstract}
Most of the modern operating systems come with accessibility and screen reading features. They also have with basic word processing applications. However, many of the languages are still not supported in the screen reading application and neither do they come with features such as translation and transliteration (phonetic). Many plugins are available to overcome these barriers, but the visually impaired users and/or users without administrative privilege will not be able to install those in the local computer. This paper discusses about implementation of a rich internet application that enables users to have access to a free online word processor which can translate, transliterate and speak out words (in different languages) that have been typed, which can be very helpful for learners of foreign languages and visually impaired users.
\end{abstract}

Keywords: languages, word processor, speech synthesis, transliteration, accessibility.

\section{Introduction}

Desktop-based applications, especially word processors are usually known to be more powerful than the online ones. They also come with more features compared to the Internet applications. However, the online applications can be very portable and can run without being installed in a computer. This paper would discuss about an online word processor which can be portable solution for many. The application can be a very important document processing utility for the visually impaired too. Some of the features would also assist learners of a new language.

There are different word processing software available in the market. Some are commercial e.g. Microsoft's Word or Apple's Pages, while others are open-source e.g. OpenOffice.org. There are also some word processors that are web browser-based Rich Internet Applications (RIA) such as Google Docs and Zoho Writer.

\section{Features Overview}

The application contains features such as automatic transliteration and translation in different languages, text-to-speech (or speech synthesis), text formatting, download or email document, keyboard controls etc. The transliteration is used for languages other 
than English, so that those who are familiar with the English keyboard can easily get the right word in a different language. This would also allow learners to know the right spelling of a word without manually checking the dictionary.

The text-to-speech speaks out the word that has been typed recently which enables the visually impaired to know if he/she typed the right word. For learners, it is also a good tool to learn the pronunciation of any specific word. The text formatting permits the users to create formal documents with proper text styles and fonts. The document is automatically saved in a user's account if the person is logged in or it is saved in the server session for that specific computer. So even if the browser crashes, the data will be saved in the server for the user to retrieve.

If the user wants to share the document, it could either be downloaded or sent by email from within the application in different formats. All of the functionalities can be accessed by using combination of keyboard keys, which is very essential for the visually impaired users.

\section{System Architecture}

The interface of the application has been built from Hypertext Markup Language (HTML), JavaScript and Asynchronous JavaScript and XML (AJAX). In the backend, PHP conducts operations such as communication between interface and text-tospeech engine, speech recognition engine; saving the document state etc.

The speech engines have been installed in a Linux server. The English text-to-speech is called Festival, developed by University of Edinburgh and the Bangla voice of Festival is developed by Center for Research on Bangla Language Processing (CRBLP), BRAC University. The translation and transliteration features will make use of the Google API, which contains a large database of words and meanings in different languages.

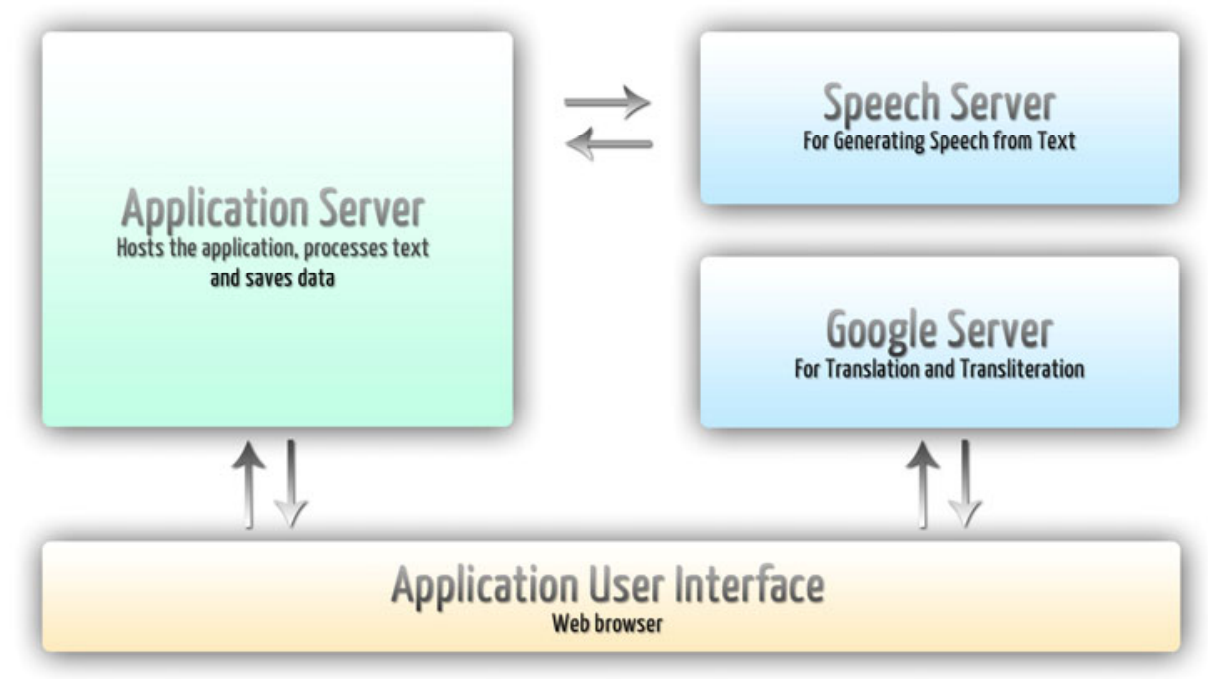

Fig. 1. The system architecture 
The JavaScript interface communicates with both the application server and Google's server. This enables a faster translation and transliteration service because the data doesn't go through the application server.

\section{User Interface}

As mentioned earlier, the Graphical User Interface (GUI) is created with HTML and JavaScript. The interface is designed with shades of gray. This can be helpful for the color-blind persons. There is a large menu button that can assist the partially visual impaired persons. The menu button can adjust the colors as well as the size of the buttons. The features that are enabled are shown in invert color, which makes a clear contrast with those who are not in use. The features can be accessed by keyboard combinations so that visually impaired can use them.

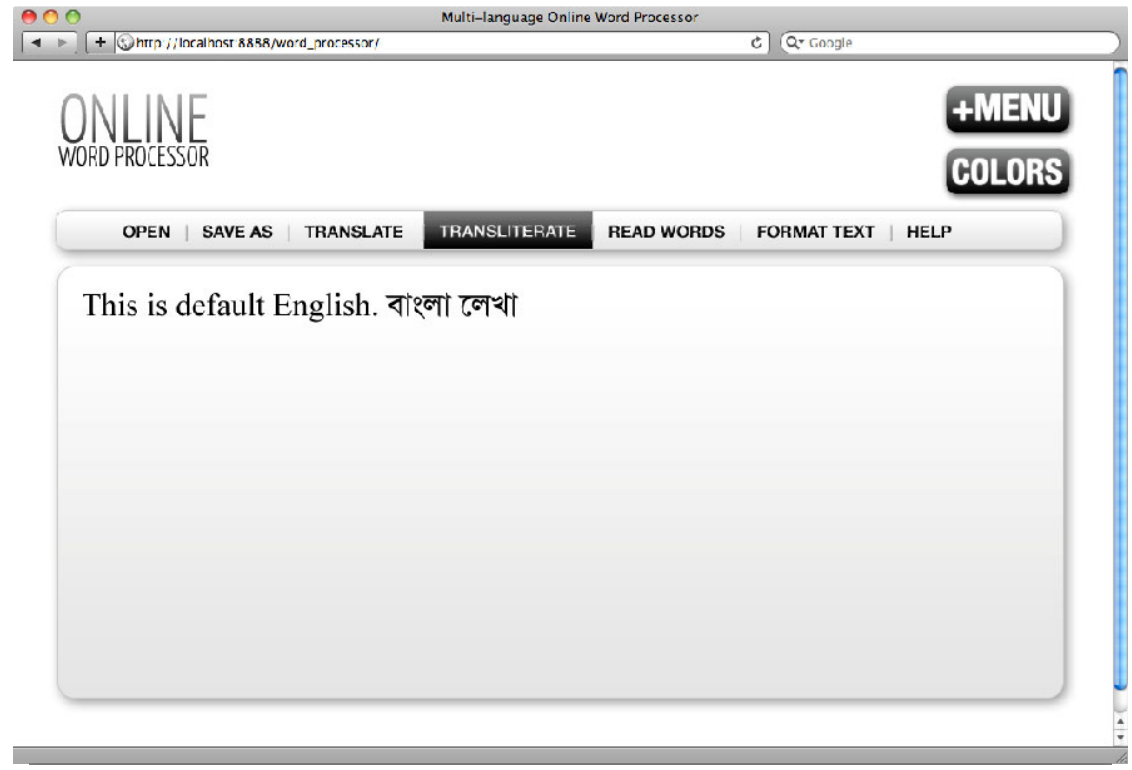

Fig. 2. Screenshot of the word processor, showing the basic functionalities and the transliteration enabled (button in black background)

\section{Speech Synthesis}

For speech synthesis or text-to-speech feature, the Festival Speech Synthesis system has been used which is open source and runs in a Linux platform. [4]

Festival offers a general framework for building speech synthesis systems as well as including examples of various modules. As a whole it offers full text to speech 
through a number APIs: from shell level, though a Scheme command interpreter, as a C++ library, from Java, and an Emacs interface. Festival is multi-lingual (currently English and Spanish). Due to being open-source, many other groups release new languages for the system including the Bangla voice by CRBLP.

\section{Translation and Transliteration}

For machine translation and phonetic transliteration, Google's services were used. Google Translate is a statistical machine translation service provided by Google Inc. to translate a section of text, document or webpage, into another language. This can be used freely by using the APIs provided by Google.

Google Translate, like other automatic translation tools, has its limitations. While it can help the reader to understand the general content of a foreign language text, it does not always deliver accurate translations. Some languages produce better results than others. However, because of being web-based, it is easier to implement it.

Google transliteration is a transliteration typing service for languages. This tool from Google is based on dictionary based phonetic transliteration approach. In contrast to older Indic typing tools (which type by transliterating under a particular scheme), it transliterates by matching the Latin words with an inbuilt dictionary. So, users do not need to remember the transliteration scheme due to which this service is very easy and suitable for first time typists or beginners.

\section{Unicode and Complex Scripts Support}

Even though many operating systems are able to display Unicode characters, most are unable to display complex glyphs if some specific fonts or programs are not installed. To overcome the missing font issue any of the following methods can be used.

\subsection{Cufón}

This is a Javascript library which can be used replace text with canvas and VML. This allows the browser to display fonts which are not installed in the system. Cufón consists of two individual parts - a font generator, which converts fonts to a proprietary format and a rendering engine written in JavaScript.

\subsection{SIFR}

The Scalable Inman Flash Replacement or sIFR is an open source JavaScript and Adobe Flash dynamic web fonts implementation, enabling the replacement of text elements on HTML web pages with Flash equivalents. It is a scalable variety of HTML text-to-flash replacement. Despite of being inside Flash, accessibility can be preserved using this technique.

Despite of having necessary fonts in the system, the system might not be able to render the fonts for complex scripts if there is no native support in the operating system. To overcome this issue, Adobe Flash can be used to properly render the glyphs of complex scripts. 
Text Layout Framework for Adobe Flash Player 10 and Adobe AIR 1.5 is an extensible library, built on the new text engine in Adobe Flash Player 10, which delivers advanced, easy-to-integrate typographic and text layout features for rich, sophisticated and innovative typography on the web. It supports bidirectional text, vertical text and over 30 writing systems including Arabic, Hebrew, Chinese, Japanese, Korean, Thai, Lao, the major writing systems of Bangladesh and India, and others.

\section{Future Developments}

Several more features have been planned for implementation in the system. They include file import/export, online spelling and grammar checking with speech synthesis etc.

\section{References}

1. Alam, F., Nath, P.K., Khan, M.: Text To Speech for Bangla Language using Festival. In: Proc. of 1st International Conference on Digital Communications and Computer Applications (DCCA 2007), Irbid, Jordan (2007)

2. Clark, R.A.J., Richmond, K., King, S.: Festival 2 - build your own general purpose unit selection speech synthesiser. In: Proc. 5th ISCA workshop on speech synthesis (2004)

3. Taylor, P.A., Black, A., Caley, R.: The architecture of the festival speech synthesis system. In: The Third ESCA Workshop in Speech Synthesis, Jenolan Caves, Australia, pp. 147-151 (1998)

4. Black., A.W., Taylor, P.A.: The Festival Speech Synthesis System: System documentation. Technical Report HCRC/TR-83, Human Communciation Research Centre, University of Edinburgh, Scotland, UK (1997),

http://www.cstr.ed.ac.uk/projects/festival.html 